\title{
POLYNOMIAL RINGS OVER A COMMUTATIVE VON NEUMANN REGULAR RING
}

\author{
ROBERT GILMER
}

\begin{abstract}
It is shown that the annihilator of each finitely generated ideal of $R\left[\left\{X_{\lambda}\right\}_{\lambda \in \Lambda}\right]$, where $R$ is a commutative von Neumann regular ring with identity, is principal; this generalizes a recent result of P. J. McCarthy.
\end{abstract}

In a recent paper [5], P. J. McCarthy showed that the ring of polynomials in one indeterminate over a commutative von Neumann regular ring $R$ with identity is semihereditary. ${ }^{1}$ Based upon results of W. Vasconcelos [8] and C. U. Jensen [3], McCarthy's proof rested upon the establishment of the following result, which we label as Theorem $A$.

Theorem A. If $f(X)=a_{0}+a_{1} X+\cdots+a_{n} X^{n}$ is in $R[X]$ and if $e_{i}$ is an idempotent generator of the ideal $a_{i} R$ for each $i$, then the annihilator of $f(X)$ is the principal ideal of $R[X]$ generated by $\left(1-e_{0}\right)\left(1-e_{1}\right) \cdots\left(1-e_{n}\right)$.

In this note we prove a result (Theorem $C$ ) that includes Theorem A; we begin with more general considerations. Let $S$ be a commutative ring, let $\left\{X_{\lambda}\right\}_{\lambda \in \Lambda}$ be a set of indeterminates over $S$, and let $f$ be an element of the polynomial ring $S\left[\left\{X_{\lambda}\right\}\right]$. If $A_{f}$ is the ideal of $S$ generated by the coefficients of $f$, then it is clear that each element of $B\left[\left\{X_{\lambda}\right\}\right]$, where $B$ is the annihilator of the ideal $A_{f}$, annihilates $f$. In the next result we give sufficient conditions in order that $B\left[\left\{X_{\lambda}\right\}\right]$ should be the annihilator of $f$.

Proposition B. ${ }^{2}$ In order that $B\left[\left\{X_{\lambda}\right\}\right]$ should be the annihilator of $f$, it is sufficient that either of the following conditions is satisfied.

(1) The ideal $A_{f}$ is idempotent.

(2) The ring $S$ contains no nonzero nilpotent element.

Proof. Assume that (1) is satisfied. Since $A_{f}$ is finitely generated, it

Received by the editors February 25, 1974. 13 C99.

AMS (MOS) subject classifications (1970). Primary 13B25, 13F 20; Secondary

$1 \mathrm{~A}$ statement of this result is also contained in [4].

$2 \mathrm{~A}$ form of this result, for power series rings, is contained in [1]; the paper [7], by J. Ohm and D. Rush, considers problems related to Proposition B. 
is principal and is generated by an idempotent element; moreover, $R$ is the direct sum of the ideals $A_{f}$ and $B$ and $R\left[\left\{X_{\lambda}\right\}\right]=A_{f}\left[\left\{X_{\lambda}\right\}\right] \oplus B\left[\left\{X_{\lambda}\right\}\right]$. Since $f$ is in $A_{f}\left[\left\{X_{\lambda}\right\}\right]$ and since the coefficients of $f$ generate $A_{f}$ as an ideal of the ring $A_{f}$ with identity, it follows from McCoy's theorem [6] that $f$ is not a zero divisor of the ring $A_{f}\left[\left\{X_{\lambda}\right\}\right]$. Consequently, $B\left[\left\{X_{\lambda}\right\}\right]$ is the annihilator of $f$, as asserted.

If $S$ contains no nonzero nilpotent element, then $(0)$ is the intersection of a family $\left\{P_{a}\right\}$ of proper prime ideals of $S$; for each $\alpha$ we denote by $\phi_{a}$ the canonical homomorphism of $S\left[\left\{X_{\lambda}\right\}\right]$ onto $\left(S / P_{\alpha}\right)\left[\left\{X_{\lambda}\right\}\right]$. If the polynomial $g$ annihilates $f$, then for each $a, \phi_{a}(f) \phi_{\alpha}(g)=0$ in $\left(S / P_{a}\right)\left[\left\{X_{\lambda}\right\}\right]$, an integral domain. Hence $\phi_{a}(f)=0$ or $\phi_{a}(g)=0$, and in any case, $g_{i} A_{f} \subseteq \bigcap_{a} P_{a}=(0)$ and $g_{i} \in B$, as we wished to prove.

If $a$ and $b$ are idempotent elements of a commutative ring $S$, then $S a \cap$ $S b=S a b$; moreover, if $U$ is an ideal of $S$, then the annihilator of $U$ is the intersection of the family of annihilators of elements of a generating set of $U$. These two elementary observations, together with Proposition B, yield Theorem $C$.

Theorem C. Let $S$ be a commutative von Neumann regular ring with identity, let $f_{1}, f_{2}, \cdots, f_{m}$ be elements of the polynomial ring $S\left[\left\{X_{\lambda}\right\}\right]$, let $\left\{a_{i}\right\}_{1}^{k}$ be the set of coefficients of the polynomials $f_{1}, f_{2}, \cdots, f_{m}$, and for each $i$ between 1 and $k$, let $e_{i}$ be an idempotent generator of the ideal $S a_{i}$ of $S$. The annihilator of the ideal ${ }^{3}$ of $S\left[\left\{X_{\lambda}\right\}\right]$ generated by $\left\{f_{j}\right\}_{j=1}^{m}$ can be described as the principal ideal of $S\left[\left\{X_{\lambda}\right\}\right]$ generated by $\left(1-e_{1}\right)\left(1-e_{2}\right)$ ... $\left(1-e_{k}\right)$ or as the principal ideal of $S\left[\left\{X_{\lambda}\right\}\right]$ generated by $1-e$, where $e$ is an idempotent generator of the ideal $\left(a_{1}, \ldots, a_{k}\right)$ of $S$.

We remark that Proposition $B$ and Theorem $C$ are true, more generally, if the polynomial ring is replaced by the semigroup ring of an arbitrary torsionfree cancellative abelian semigroup with zero. Moreover, an even shorter proof of Proposition B can be obtained by the application of the DedekindMertens lemma, which is known to generalize to such semigroup rings.

\section{REFERENCES}

1. R. Gilmer, A. Grams and T. Parker, Zero divisors in power series rings, J. Reine Angew. Math. (to appear).

2. R. Gilmer and T. Parker, Semigroup rings as Prüfer rings, Duke Math. J. 41 (1974), 219-230.

3If $\left|\left\{X_{\lambda}\right\}\right|=1$, then each finitely generated ideal of $S\left[\left\{X_{\lambda}\right\}\right]$ is principal [2], but this is not the case, of course, for polynomial rings in more than one variable. 
3. C. U. Jensen, On homological dimensions of rings with countably generated ideals, Math. Scand. 18 (1966), 97-105. MR 34 \#7611.

4. P. LeBihan, Sur la cohérence des anneaux de dimension homologique 2, C. R. Acad. Sci Paris Sér. A-B 273 (1971), A342-A345. MR 46 \#5043.

5. P. J. McCarthy, The ring of polynomials over a von Neumann regular ring, Proc. Amer. Math. Soc. 39 (1973), 253-254. MR 47 \#5043.

6. N. H. McCoy, Remarks on divisors of zero, Amer. Math Monthly 49 (1942), 286-295. MR 3, 262.

7. J. Ohm and D. E. Rush, Content modules and algebras, Math. Scand. 31 (1972), 49-68.

8. W. V. Vasconcelos, On finitely generated flat modules, Trans. Amer. Math. Soc. 138 (1969), 505-512. MR 39 \#199.

DEPARTMENT OF MATHEMATICS, FLORIDA STATE UNIVERSITY, TALLAHASSE, FLORIDA 32306 (Current address)

DEPARTMENT OF MATHEMATICS, LA TROBE UNIVERSITY, BUNDOORA, VICTORIA 3083, AUSTRALIA 